\title{
ИДЕНТИФИКАЦИЯ ОБЪЕКТОВ СО СЛУЧАЙНЫМ ДРЕЙФОМ ПАРАМЕТРОВ
}

Адаптация модели к изменениям свойств промышленных объектов - важная для практики задача. В зависимости от наличия или отсутствия прогноза дрейфа возможны два подхода к решению задачи. Алгоритм, разработанный в [ $\left.{ }^{1}\right]$, предполагает детерминированный характер дрейфа и является весьма чувствительным к точности задания траектории $\left[{ }^{2}\right]$. Однако дрейф промышленных объектов имеет, как правило, стохастический характер. В данной статье выводится новый алгоритм адаптации модели объекта к дрейфу с заданными вероятностными закономерностями.

\section{1. Постановка задачи}

Пусть интересующий нас объект описывается в дискретные моменты времени $t=0,1, \ldots, n, \ldots$ линейным по параметрам уравнением

$$
v_{n}=z_{n}^{\mathrm{T}} \beta_{n},
$$

где $v_{n}, z_{n}$ и $\beta_{n}$ - выход, векторы входа и коэффициентов объекта в момент времени $n$ соответственно. Последовательность $\left\{\beta_{t}\right\}$ рассматривается как пространственно независимый, обобщенно-стационарный временной ряд, описываемый в момент времени $n$ параметрической моделью $\left[{ }^{3}\right]$ вида

$$
\begin{aligned}
& \beta_{n}=\tilde{\beta}_{n}+\mu, \quad \tilde{\beta}_{n}=\tilde{\beta}_{n-1}(1)+\alpha_{n}, \\
& \tilde{\beta}_{n-1}(1)=\sum_{v=1}^{l} \varphi_{v} \tilde{\beta}_{n-v}-\sum_{v=1}^{q} \Theta_{v} \alpha_{n-v} .
\end{aligned}
$$

Здесь $\mu$ - безусловное среднее временного ряда; $\beta_{n-1}(1)-$ прогноз дрейфа с момента $n-1$ на один шаг вперед; $\alpha_{n}$ - гауссов белый шум в момент $n$ с нулевым средним и дисперсией $D ; \Theta_{v}$ и $\varphi_{v}-$ матрицы параметров оператора скользящего среднего $\Theta(B)$ порядка $q$ и нестационарного оператора авторегрессии $\varphi(B)$ порядка $l$ при сдвиге назад на $v$. Применяемые нами матрицы параметров $\pi_{v}$ и $\Psi_{v}$ являются элементами операторов

$$
\pi(B)=\varphi^{-1}(B) \Theta(B), \quad \Psi(B)=\pi^{-1}(B) .
$$

Допускается, что ошибка измерения выхода $h_{n}$ обладает свойством аддитивности с неизменным нормальным распределением

$$
y_{n}=v_{n}+h_{n}, \quad h_{n} \sim N_{1}(0, r) .
$$


Величины $h_{n}, z_{n}$ и $\beta_{n}$ принимаются попарно-независимыми. Қвадратичная функция потерь выбирается в виде

$$
W\left(b_{n}, \beta_{n}\right)=\left[b_{n}-\beta_{n}\right]^{\mathrm{T}}\left[b_{n}-\beta_{n}\right],
$$

где $b_{n}$ - условная оценка вектора истинных параметров $\beta_{n}$ в текущий момент времени.

Ставится задача синтеза оптимального в смысле минимума полного риска

$$
R=M\left\{\sum_{n=0}^{N} W\left(b_{n}, \beta_{n}\right)\right\}
$$

алгоритма оценки нензвестных дрейфующих параметров при ограничении, задаваемом его физической реализуемостью.

\section{2. Алгоритм оценки дрейфующих параметров объекта}

Согласно факторизационному критерию $\left[{ }^{4}\right]$, прогноз дрейфа $\boldsymbol{\beta}_{n-1}(1)$ является достаточной статистикой параметра $\beta_{n}$ в момент $n-1$. Поступлением текущих наблюдений от входа и выхода объекта прогноз $\beta_{n-1}(1)$ уточняется. Таким образом, полный риск предсказания параметров объекта выражается в виде

$$
R=\sum_{n=0}^{N} \int_{\Omega(\cdot)} W\left(b_{n}, \beta_{n}\right) P\left(b_{n}, \beta_{n} / y_{n}, z_{n}, \beta_{n-1}(1)\right) P\left(y_{n}, z_{n}, \beta_{n-1}(1)\right) d \Omega,
$$

где $P(\cdot)$ - безусловное распределение величин $y_{n}, z_{n}$ и $\beta_{n-1}(1)$; $P(\cdot / \cdot)$ - условное распределение векторов $b_{n}$ и $\beta_{n}$ при фиксированных $y_{n}, z_{n}, \beta_{n-1}(1) ; \Omega(\cdot)$ - область ннтегрирования по $b_{n}, \boldsymbol{\beta}_{n}, y_{n}, z_{n}$ и $\beta_{n-1}(1)$.

Нетрудно показать, что найденная минимизацией функционала (7) оценка параметров объекта

$$
b_{n}^{*}=b_{n}^{*}\left(y_{n}, z_{n}, \beta_{n-1}(1)\right)
$$

имеет ряд оптимальных свойств (несмещенность, достаточность, эффективность и т. д.) и простой алгоритм вычисления. Однако оценка эта физически не реализуема, поскольку данные о предыстории дрейфа $\beta_{n-1}, \beta_{n-2}, \ldots$, необходимые для вычисления прогноза $\beta_{n-1}(1)$, как правило, отсутствуют.

Чтобы получить реализуемую стратегию, вместо прогноза реальной предыстории $\beta_{n-1}(1)$ следует использовать его оценку $b_{n-1}(1)$, найденную с помощью оценок дрейфа на предыдущих тактах

$$
\begin{gathered}
b_{n-1}(1)=\sum_{v=1}^{l} \varphi_{v} \tilde{b}_{n-v}-\sum_{v=1}^{q} \Theta_{v} a_{n-v}+\mu, \\
b_{t}=\left\{\begin{array}{ll}
b_{t}^{\mathrm{H}}, & t<0, \\
b_{t}+\mu, & t \geqslant 0,
\end{array} \quad a_{t}= \begin{cases}a_{t}^{\mathrm{H}}, & t<0, \\
b_{t}-b_{t-1}(1), & t \geqslant 0,\end{cases} \right.
\end{gathered}
$$

где $b_{t}{ }^{\mathrm{H}}, a_{t}{ }^{\mathrm{H}}-$ определенным образом восстановленные начальные условия разностного уравнения (см. раздел 3), а затем произвести дополнительное усреднение в (7) по разности $\beta_{n-1}(1)-b_{n-1}(1)$, состоящей из двух компонент: ощибки обучения $\Delta_{n}{ }^{\text {об }}$ н ошибки отслеживания 
$\Delta_{n}{ }^{\text {от. }}$. Первую порождает неопределенность начальных условий $b_{t^{\mathrm{H}}}, a_{t^{\mathrm{H}}}$ $(t<0)$, а вторую - неточное отслеживание дрейфа на предыдущих тактах.

Записав полученный в результате искажения $\beta_{n-1}(1)$ ошибкой $\Delta_{n}{ }^{\text {oб }}$ вектор прогноза в виде

$$
\begin{aligned}
& b_{n-1}^{\mathrm{o \sigma}}(1)=\sum_{v=1}^{l} \varphi_{v} \tilde{b}_{n-v}^{\mathrm{o \sigma}}-\sum_{v=1}^{q} \Theta_{v} a_{n-v}^{\mathrm{o \sigma}}, \\
& b_{t}^{\mathrm{o \sigma}}=\left\{\begin{array}{ll}
b_{t}^{\mathrm{H}}, & t<0, \\
\beta_{t}, & t \geqslant 0,
\end{array} a_{t}^{\mathrm{o \sigma}}= \begin{cases}a_{t}^{\mathrm{H}}, & t<0, \\
b_{t}^{\mathrm{o \sigma}}-b_{t-1}^{\mathrm{o \sigma}}(1), & t \geqslant 0,\end{cases} \right.
\end{aligned}
$$

определим суммарную ошибку

$$
\begin{gathered}
\Delta_{n}=\Delta_{n}^{\text {oб }}+\Delta_{n}^{\text {oT }}=\beta_{n-1}(1)-b_{n-1}(1)= \\
=\left[\beta_{n-1}(1)-b_{n-1}^{\text {oб }}(1)\right]+\left[b_{n-1}^{\text {oб }}(1)-b_{n-1}(1)\right] .
\end{gathered}
$$

Согласно выражению (7), усреднение риска $R$ по ошибкам (11) дает

$$
R=\sum_{\substack{n=0 \Omega\left(b_{n}, \beta_{n}, \beta_{n-1}(1),\left(b_{n-1}^{\circ \sigma}(1), y_{n}, z_{n}, b_{n-1}(1)\right)\right.}} W\left(b_{n}, \beta_{n}\right) P\left(b_{n}, \beta_{n}, \beta_{n-1}(1), b_{n-1}^{\text {of }}(1) / y_{n}, z_{n}, b_{n-1}(1)\right) \times
$$$$
\times P\left(y_{n}, z_{n}, b_{n-1}(1)\right) d \Omega \text {. }
$$

В Приложении доказывается, что алгоритм оценки дрейфующих параметров объекта, минимизирующий полный риск (12), имеет вид

$$
\begin{aligned}
& b_{t}=b_{t-1}(1)+\Gamma_{t} z_{t}\left[y_{t}-z_{t}^{\mathrm{T}} b_{t-1}(1)\right], \\
& \Gamma_{t}=\left[r+z_{t}^{\mathrm{T}} K_{t} z_{t}\right]^{-1} K_{t}, \\
& K_{t}=D+D_{t}^{\mathrm{oT}}+D_{t}^{\mathrm{o \sigma}} \\
& D_{t}^{\mathrm{oT}}=\sum_{v=1}^{t} \pi_{v} D_{t-v} \pi_{v}^{\mathrm{T}} \\
& D_{t}=r \Gamma_{t}, \quad t=0,1,2, \ldots
\end{aligned}
$$

Здесь $b_{t-1}(1)-$ оценка параметра $\beta_{t}$ с момента $t-1$,на один шаг вперед, определяемая формулами (9); $\Gamma_{t}-$ шаг коррекции; $K_{t}$ и $D_{t}-$ дисперсии оценок $b_{t-1}(1)$ и $b_{t} ; D_{t}{ }^{\circ \sigma}$ и $D_{t}$ от - дисперсии ошибок обучения и отслеживания соответственно. $D_{t}{ }^{\text {об }}$ определяется по формуле (П 12).

В силу нестационарности объекта шаг коррекции параметров, а также дисперсия ошибок отслеживания к нулю не стремятся. Дисперсия же ошибок обучения по мере устранения начальной неопределенности, напротив, приближается к нулю. В зависимости от степени значимости ошибок обучения по сравненню с ошибками отслеживания работа алгоритма разделяется на два режима - обучения и отслеживания. Иногда в связи с кратковременностью режима обучения и отсутствием требования оптимальности работы алгоритма разумно начиная с первых тактов отказаться от точного учета ценности априорной информации и применить для расчета упрощенные формулы в пределе $D_{t}{ }^{\mathrm{o \sigma}}=0$. 


\section{3. Восстановление начальных условий}

При точном задании начальных условий $\left[b_{t}{ }^{\mathrm{H}}=\beta_{t}, t=[-l,-1]\right\}$, $\left\{a_{t}{ }^{\mathrm{H}}=\alpha_{t}, t=[-q,-1]\right\}$ режим обучения в работе алгоритма отсутствует. В практике такая ситуация не реальна, т. е. надобность в разумном выборе начальных условий существует всегда.

Импульсы белого шума предсказать нельзя, поэтому априорные оценки $\left\{a_{t^{\mathrm{H}}}, t=[-q,-1]\right\}$ приравниваются к их безусловным математическим ожиданиям

$$
a_{t}^{\mathrm{H}}=M\left\{\alpha_{t}\right\}=0 .
$$

Априорные значения дрейфующих коэффициентов $\left\{b_{t}{ }^{\mathrm{H}}, t=[-l,-1]\right\}$ могут также восстанавливаться приравниванием к безусловному математическому ожиданию временного ряда $\left\{\beta_{t}\right\}$

$$
b_{t}^{\mathrm{H}}=M\left\{\beta_{t}\right\}=\mu .
$$

Достоинством такого приближения является отсутствие надобности в наблюдениях по оцениванию начальных условий, недостатком - грубость оценок, если дрейф по характеру близок к нестационарному процессу.

\section{4. Пример}

Продемонстрируем адаптацию модели на одномерном имитационном объекте со спецификацией

$$
\begin{gathered}
y_{t}=\beta_{0 t}+z_{1 t} \beta_{1 t}+h_{t}, \\
\beta_{0 t}=-0,5 \tilde{\beta}_{0 t-1}-0,5 \alpha_{0 t-1}+\alpha_{0 t}+2, \\
\beta_{1 t}=0,7 \beta_{1 t-1}+\alpha_{1 t}+1, \\
h_{t}, \alpha_{1 t} \sim N_{1}(0,0.15), \alpha_{0 t} \sim N_{1}(0,0.25), z_{1 t} \sim N_{1}(2,1) .
\end{gathered}
$$

На рис. 1 и 2 непрерывными линиями изображены реализации дрейфа наклона $\left\{\beta_{1 t}, t=0, \ldots, 50\right\}$ и аддитивного шума $\left\{\beta_{0 t}, t=0, \ldots, 50\right\}$. Соответствующие оценки параметров, найденные с помощью алгоритма (13), показаны пунктирными линиями.

Проведенный статистический анализ неувязок

$$
e_{t}=v_{t}-z_{t}^{\mathrm{T}} b_{t}
$$

не обнаружил значимого отклонения выборочного среднего от нуля и временной корреляции между неувязками. Несмотря на сравнительно высокий уровень шумов, алгоритм успешно отслеживает стохастически дрейфующие коэффициенты, что недостижимо другими известными методами.

\section{ПРИЛОЖЕНИЕ}

\section{Вывод алгоритма оценки дрейфующих параметров объекта}

Полный риск предсказания параметров объекта $R$ выражается через сумму удельных рисков $R_{n}$ :

$$
R=M\left\{\sum_{n=0}^{N} W\left(b_{n}, \beta_{n}\right)\right\}=\sum_{n=0}^{N} M\left\{W\left(b_{n}, \beta_{n}\right)\right\}=\sum_{n=0}^{N} R_{n},
$$




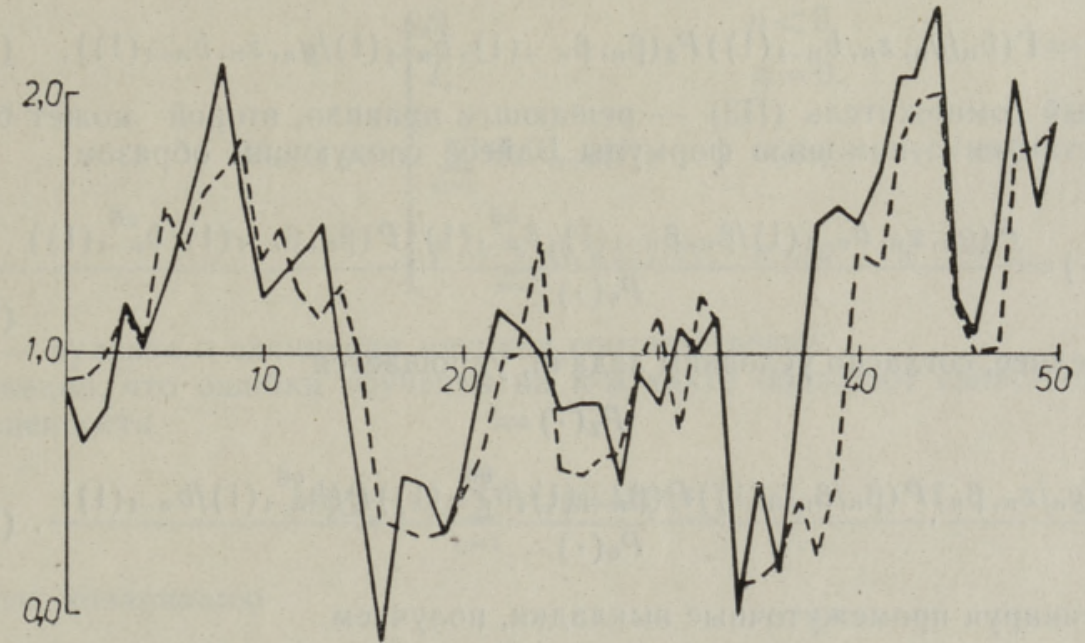

Рис. 1. Дрейф параметра наклона $\beta_{1 t}$ и его оценка $b_{1 t}$.

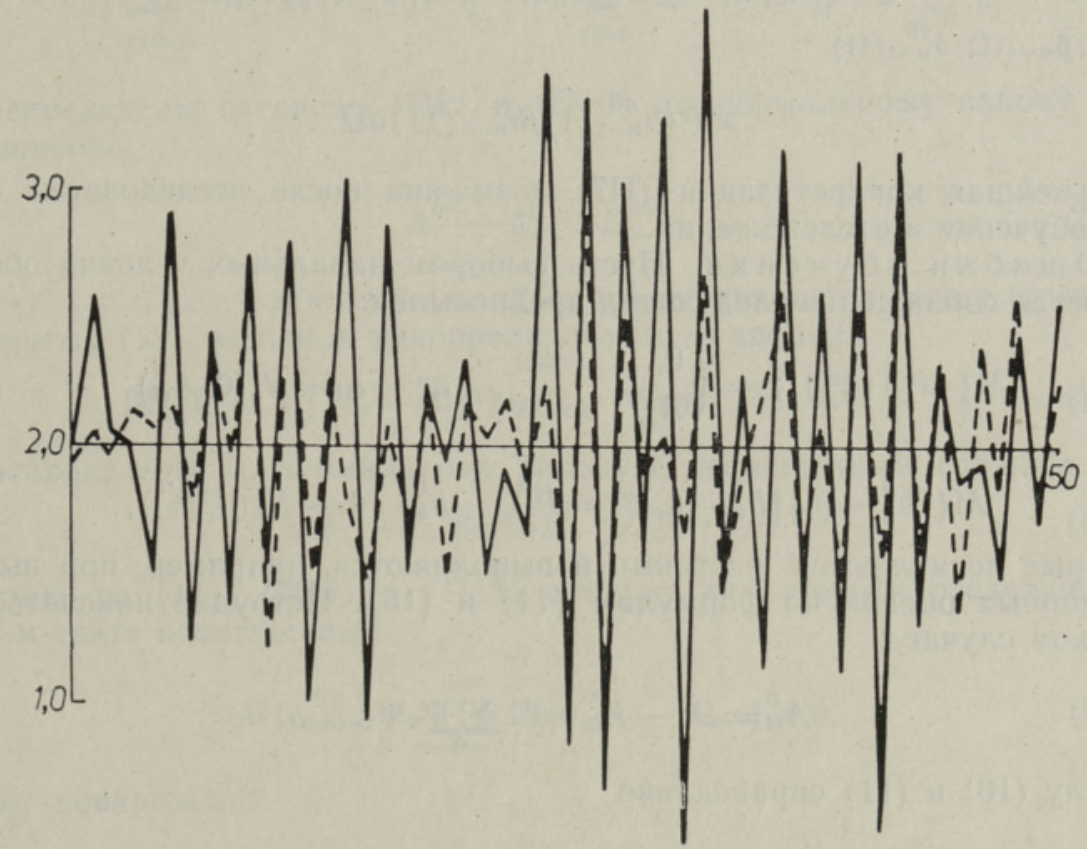

Рис. 2. Дрейф аддитивного шума $\beta_{0 t}$ и его оценка $b_{0 t}$.

где $R_{n}$, согласно (12), имеет вид

$$
\begin{gathered}
R_{n}=\int_{\Omega} W\left(b_{n}, \beta_{n}\right) P_{1}\left(b_{n}, \beta_{n}, \beta_{n-1}(1), b_{n-1}^{0 \sigma}(1) / y_{n}, z_{n}, b_{n-1}(1)\right) \times \\
\times P_{0}\left(y_{n}, z_{n}, b_{n-1}(1)\right) d \Omega .
\end{gathered}
$$

Применение теоремы умножения вероятностей ко второму сомножителю подынтегрального выражения дает 
$P_{1}(\cdot)=\Gamma\left(b_{n} / y_{n}, z_{n}, b_{n-1}(1)\right) P_{2}\left(\beta_{n}, \beta_{n-1}(1), b_{n-1}^{o \sigma}(1) / y_{n}, z_{n}, b_{n-1}(1)\right)$.

Первый сомножитель (П3) - решающее правило, второй может быть представлен с помощью формулы Байеса следующим образом:

$$
P_{2}(\cdot)=\frac{P\left(y_{n}, z_{n}, b_{n-1}(1) / \beta_{n}, \beta_{n-1}(1), b_{n-1}^{\text {oб }}(1)\right)}{P_{0}(\cdot)} \frac{P\left(\beta_{n}, \beta_{n-1}(1), b_{n-1}^{\text {oб }}(1)\right)}{\text { (П4) })} .
$$

Последнее, согласно условиям задачи, упрощается

$$
\begin{gathered}
P_{2}(\cdot)= \\
=\frac{P\left(y_{n} / z_{n}, \beta_{n}\right)}{P} \cdot \frac{P\left(\beta_{n} / \beta_{n-1}(1)\right) P\left(\beta_{n-1}(1) / b_{n-1}^{\text {oб }}(1)\right) P\left(b_{n-1}^{\text {oб }}(1) / b_{n-1}(1)\right)}{P_{0}(\cdot)} .
\end{gathered}
$$

Комбинируя промежуточные выкладки, получаем

$$
R_{n}=\int_{\Omega\left(y_{n}, b_{n}\right)} x_{n} \Gamma\left(b_{n} / y_{n}, z_{n}, b_{n-1}(1)\right) d \Omega,
$$

$$
\begin{aligned}
& x_{n}=\int W\left(b_{n}, \beta_{n}\right) P\left(y_{n} / z_{n}, \beta_{n}\right) P\left(\beta_{n} / \beta_{n-1}(1)\right) P\left(\beta_{n-1}(1) / b_{n-1}^{\text {oб }}(1)\right) \times \\
& \Omega\left(\beta_{n}, \beta_{n-1}(1), b_{n-1}^{06}(1)\right)
\end{aligned}
$$

$$
\times P\left(b_{n-1}^{\text {o6 }}(1) / b_{n-1}(1)\right) d \Omega .
$$

Дальнейшая конкретизация (П7) возможна после исследования ошибок обучения и отслеживания.

Ош и бк и обучения. Пусть выбором начальных условий обеспечивается соблюдение следующих предпосылок:

$$
\begin{aligned}
& M\left\{\left(a_{t}^{\mathrm{H}}\right)\left(a_{s}^{\mathrm{H}}\right)^{\mathrm{T}}\right\}=\left\{\begin{array}{ll}
0, & t \neq s \\
A_{t t}^{\mathrm{H}}, & t=s
\end{array}, \quad a_{t}^{\mathrm{H}}-\alpha_{t} \sim N_{\ni}\left(0, A_{t t}^{\mathrm{H}}\right),\right. \\
& M\left\{\left[b_{t}^{\mathrm{H}}-\beta_{t}\right]\left[b_{s}^{\mathrm{H}}-\beta_{s}\right]^{\mathrm{T}}\right\}=P_{t s}^{\mathrm{H}}, \quad b_{t}^{\mathrm{H}}-\beta_{t} \sim N_{\ni}\left(0, P_{t t}^{\mathrm{H}}\right),
\end{aligned}
$$

которые не являются жесткими и выполняются, например, при выборе априорных оценок по формулам (14) и (15). Нетрудно показать, что в таком случае

$$
A_{t t}^{\mathrm{H}}=D, \quad P_{t s}^{\ddot{*}}=D \sum_{v=0}^{\infty} \Psi_{v} \Psi_{v-(s-t)}^{\mathrm{T}} .
$$

В силу (10) и (11) справедливо

$$
\Delta_{n}^{\mathrm{o \sigma}}=\sum_{v=1}^{l} \varphi_{v}\left(b_{n-v}^{0 \sigma}-\beta_{n-v}\right)-\sum_{v=1}^{q} \Theta_{v}\left(a_{n-v}^{0 \sigma}-\alpha_{n-v}\right) .
$$

Пропуская несложные, но громоздкие преобразования и привлекая метод математической индукции, выразим ошибку обучения в текущий момент через начальные условия (для случая $l=q$ ):

$$
\Delta_{n}^{\mathrm{o \sigma}}=\sum_{t=1}^{q} V_{n}(t) \Delta_{-t}^{\mathrm{H}} \text {, }
$$

где

$$
\Delta_{-t}^{\mathrm{H}}=\varphi_{t}\left(b_{-t}^{\mathrm{H}}-\beta_{-t}\right)-\Theta_{t}\left(a_{-t}^{\mathrm{H}}-\alpha_{-t}\right),
$$




$$
V_{n}(t)= \begin{cases}0, & n<0, \\ I, & n=0, \\ \sum_{s=1}^{q} \Theta_{s} V_{n-s}(t), & n \geqslant q \quad \text { или } t=q, \\ I-\sum_{s=1}^{q-t} \Theta_{s} V_{n-s}(t), & n<q \quad \text { и } \quad t \neq q,\end{cases}
$$

$0, I$ - нулевая и единичная матрица соответственно.

Очевидно, что ошибки обучения на $n$-м такте обладают свойством несмещенности

$$
M\left\{\Delta_{n}^{\mathrm{o} \sigma}\right\}=\sum_{t=1}^{q} V_{n}(t) M\left\{\Delta_{-t}^{\mathrm{H}}\right\}=0,
$$

имеют ковариацию

$$
\begin{gathered}
D_{n}^{\mathrm{o} \sigma}=M\left\{\left(\Delta_{n}^{\mathrm{o \sigma}}\right)\left(\Delta_{n}^{\mathrm{o} \sigma}\right)^{\mathrm{T}}\right\}=\sum_{s, t=1}^{q} V_{n}(t) M\left\{\left(\Delta_{-i}^{\mathrm{H}}\right)\left(\Delta_{-s}^{\mathrm{H}}\right)^{\mathrm{T}}\right\} V_{n}^{\mathrm{T}}(s)= \\
=\sum_{s, t=1}^{q} V_{n}(t)\left[\varphi_{t} P_{t s}^{\mathrm{H}} \varphi_{s}^{\mathrm{T}}\right] V_{n}^{\mathrm{T}}(s)+\sum_{t=1}^{q} V_{n}(t)\left[\Theta_{t} A_{t t}^{\mathrm{H}} \Theta^{\mathrm{T}}\right] V_{n}^{\mathrm{T}}(t)
\end{gathered}
$$

и распределены согласно (П8) и (П10) по нормальному закону распределения

$$
\Delta_{n}^{\text {об }} \sim N_{\ni}\left(0, D_{n}^{\text {об }}\right)
$$

О ш и б к и о т с л е ж в а н я. Доказуемо, что найденные с помощью алгоритма (13) оценки $b_{t}$ некоррелированы во времени

$$
M\left\{\left[b_{t}-\beta_{t}\right]\left[b_{s}-\beta_{s}\right]^{\mathrm{T}}\right\}=0 \quad \forall s \neq t, s, t=0,1, \ldots
$$

и распределены по нормальному закону с несмещенным средним

$$
b_{t} \sim N_{\ni}\left(\beta_{t}, D_{t}\right), \quad D_{t}=r \Gamma_{t} .
$$

Поэтому из (11), (П14) и (П15) следует, что ошибки отслеживания на $n$-м такте несмещенные

$$
M\left\{\Delta_{n}^{\text {от }}\right\}=\sum_{v=1}^{n} \pi_{v} M\left\{b_{n-v}^{\text {об }}-b_{n-v}\right\}=0,
$$

имеют ковариацию

$$
\begin{gathered}
D_{n}^{\mathrm{oT}}=M\left\{\left(\Delta_{n}^{\mathrm{oT}}\right)\left(\Delta_{n}^{\mathrm{oT}}\right)^{\mathrm{T}}\right\}= \\
\sum_{v=1}^{n} \pi_{v} M\left\{\left[b_{n-v}^{\mathrm{o \sigma}}-b_{n-v}\right]\left[b_{n-v}^{\mathrm{o \sigma}}-b_{n-v}\right]^{\mathrm{T}} \pi_{v}^{\mathrm{T}}\right\}= \\
=\sum_{v=1}^{n} \pi_{v} D_{n-v} \pi_{v}^{\mathrm{T}}
\end{gathered}
$$

и распределены по нормальному закону распределения

$$
\Delta_{n}^{\mathrm{oT}} \sim N_{\ni}\left(0, D_{n}^{\mathrm{oT}}\right)
$$

Уравнение (П7) может быть конкретизировано с учетом свойств ошибок и условий задачи. Получим 


$$
\begin{gathered}
x_{n}=c^{-1 / 2} \int_{-\infty}^{\infty} \int_{-\infty}^{\infty} \int_{-\infty}^{\infty}\left[b_{n}-\beta_{n}\right]^{\mathrm{T}}\left[b_{n}-\beta_{n}\right] \exp \left\{-\frac{1}{2}\left[\beta_{n}-\beta_{n-1}(1)\right]^{\mathrm{T}} D^{-1} X\right. \\
\times\left[\beta_{n}-\beta_{n-1}(1)\right]-\frac{1}{2 r}\left[y_{n}-z_{n}^{\mathrm{T}} \beta_{n}\right]^{2}-\frac{1}{2}\left[\beta_{n-1}(1)-b_{n-1}^{\text {об }}(1)\right]^{\mathrm{T}}\left(D_{n}^{\text {oT }}\right)^{-1} X \\
\times\left[\beta_{n-1}(1)-b_{n-1}^{\text {oб }}(1)\right]-\frac{1}{2}\left[b_{n-1}^{\text {об }}(1)-b_{n-1}(1)\right]^{\mathrm{T}}\left(D_{n}^{\text {oT }}\right)^{-1} \times \\
\left.\times\left[b_{n-1}^{\text {об }}(1)-b_{n-1}(1)\right]\right\} d \beta_{n} d \beta_{n-1}(1) d b_{n-1}^{\text {об }}(1), \\
c=(2 \pi)^{3 э+1} r|D|\left|D_{n}^{\text {oб }}\right|\left|D_{n}^{\text {от }}\right|,
\end{gathered}
$$

где э - размерность объекта.

Решающее правило на $n=N$-м такте следовало бы определить из условия минимума удельного риска $R_{n=N}$, но в силу регулярности оптимальной байесовской стратегии $\left[{ }^{5}\right]$, оно определяется из условия минимума функционала (П19).

Решение интеграла (П19), согласно [6], выражается в виде

$$
\begin{gathered}
\varkappa_{n}=\left\{\left[b_{n}-\varepsilon_{n}^{-1}\left(r\left(b_{n-1}(1)-\mu\right)+K_{n} z_{n}\left(y_{n}-z_{n}^{\mathrm{T}} \mu\right)\right)\right]^{2}+\varepsilon_{n}^{-1} r K_{n}\right\} \times \\
\times \varepsilon_{n}^{-1 / 2} \exp \left\{-\frac{1}{2} \varepsilon_{n}^{-1}\left(y_{n}-z_{n}^{\mathrm{T}} b_{n-1}(1)\right)^{2}\right\},
\end{gathered}
$$

где $\varepsilon_{n}=r+z_{n}^{\mathrm{T}} K_{n} z_{n}, \quad K_{n}=D+D_{n}^{\text {от }}+D_{n}^{\text {об }}$.

В результате минимизации последнего получим правило уточнения оценок на $(n=N)$-м такте

$$
b_{n}=b_{n-1}(1)+\Gamma_{n} z_{n}\left(y_{n}-z_{n} b_{n-1}(1)\right), \quad \Gamma_{n}=\varepsilon_{n}^{-1} K_{n} .
$$

Нетрудно видеть, что рассматриваемая система нейтральна, поскольку

$$
R_{n}=\int_{-\infty}^{\infty} x_{n}^{*} d y_{n}=\left|\varepsilon_{n}^{-1} r K_{n}\right|,
$$

где $x_{n}{ }^{*}$ - минимальное значение функции (П20) по $b_{n}$, поэтому алгоритм уточнения (П21) во времени $t=0,1, \ldots, n, \ldots$ не изменяетcя $\left[{ }^{5}\right]$.

\section{ЛИ Т Е РА Т У Р А}

1. Цы пкин Я. 3., К а плин ский А. И., Л а ринов К. А., Техническая кибернетика, № 5, 9 (1970).

2. В ел е в К. Д., Автоматика и телемеханика, № 8, 40 (1975).

3. Бокс Дж., Д жен ки н с Г., Анализ временных рядов. Прогноз и управление, М., 1974.

4. Де Г ро о т М., Оптимальные статистические решения, М., 1974.

5. Фельдб а ум А. А., Основы теории оптимальных автоматических систем, М., 1966.

6. Гр адш тей н И. С., Р ыжик И. М., Таблицы интегралов, сумм, рядов и произведений, М., 1962.

Научно-исследовательский

и проектно-технологический институт

Таллинского электротехнического завода

им. М. И. Калинина 
R. TENNO

\section{JUHUSLIKULT MUUTUVATE PARAMEETRITEGA OBJEKTIDE IDENTIFITSEERIMINE}

Artiklis on käsitletud statistilise otsustusteooria kasutamist objekti juhuslikult ajas muutuvate parameetrite hindamiseks. On analüüsitud juhtu, kus objekti väljund on mõõdetud aditiivse müraga, ning esitatud mudeli adapteerimise algoritm ja teoreetilisi seisukohti. illustreeriv näide.

\section{R. TENNO}

\section{IDENTIFICATION OF PLANTS HAVING STOCHASTIC TIME-VARYING PARAMETERS}

The application of the theory of statistical decisions to problems of estimating stochastic time-varying parameters of plants is discussed. A case is analysed when the plant output is measured with a random additive noise. The author proposes an adaption algorithm of the model. The theoretical assertions are illustrated by an example. 